\title{
BMJ Open Delay in the induction of labour process: a retrospective cohort study and computer simulation of maternity unit workload
}

\author{
Katherine Robertson (D , ${ }^{1}$ lan Hardingham, ${ }^{2}$ Rhiannon D'Arcy, ${ }^{3}$ Aparna Reddy, ${ }^{3}$ \\ Joe Clacey ${ }^{4}$
}

To cite: Robertson $\mathrm{K}$, Hardingham I, D'Arcy R, et al. Delay in the induction of labour process: a retrospective cohort study and computer simulation of maternity unit workload. BMJ Open 2021;11:e045577. doi:10.1136/ bmjopen-2020-045577

- Prepublication history and additional supplemental material for this paper are available online. To view these files, please visit the journal online (http://dx.doi.org/10.1136/ bmjopen-2020-045577).

Received 06 0ctober 2020 Accepted 11 June 2021

\section{Check for updates}

\section{(c) Author(s) (or their} employer(s)) 2021. Re-use permitted under CC BY-NC. No commercial re-use. See rights and permissions. Published by BMJ.

${ }^{1}$ Department of Obstetrics \& Gynaecology, Oxford University Hospitals NHS Foundation Trust, Oxford, UK

${ }^{2}$ Oxford Brain Diagnostics,

Oxford, UK

${ }^{3}$ Department of Obstetrics \& Gynaecology, Buckinghamshire Healthcare NHS Trust, Aylesbury, UK

${ }^{4}$ Department of Child Psychiatry, Oxford Health NHS Foundation Trust, Oxford, UK

Correspondence to Dr Katherine Robertson; katherine.robertson@ouh.nhs.uk

\section{ABSTRACT}

Objectives Delay in the induction of labour (IOL) process is associated with poor patient experience and adverse perinatal outcome. Our objective was to identify factors associated with delay in the IOL process and develop interventions to reduce delay.

Design and settings We performed a retrospective cohort study of maternity unit workload in a large UK district general hospital. Electronic hospital records were used to quantify delay in the IOL process and linear regression analysis was performed to assess significant associations between delay and potential causative factors. A novel computer maternity unit simulation model, MUMSIM (Maternity Unit Management SIMulation), was developed using real-world data and interventions were tested to identify those associated with a reduction in delay. Participants All women giving birth at Stoke Mandeville Hospital, Buckinghamshire National Health Service (NHS) Trust in $2018(\mathrm{n}=4932)$.

Primary outcome measure Delay in the $10 \mathrm{~L}$ process of more than 12 hours.

Results The retrospective analysis of real-world maternity unit workload showed $30 \%$ of women had IOL and of these, $33 \%$ were delayed $>12$ hours with $20 \%$ delayed $>24$ hours, $10 \%$ delayed $>48$ hours and $1.3 \%$ delayed $>72$ hours. Delay was significantly associated with the total number of labouring women $(p=0.008)$ and the number of booked IOL $(p=0.009)$ but not emergency IOL, spontaneously labouring women or staffing shortfall. The MUMSIM computer simulation predicted that changing from slow release 24-hour prostaglandin to 6-hour prostaglandin for primiparous women would reduce delay by $4 \%(p<0.0001)$ and that additional staffing interventions could significantly reduce delay up to $17.9 \%$ $(p<0.0001)$.

Conclusions Planned obstetric workload of booked IOL is associated with delay rather than the unpredictable workload of women in spontaneous labour or emergency IOL. We present a novel maternity unit computer simulation model, MUMSIM, which allows prediction of the impact of interventions to reduce delay.

\section{INTRODUCTION}

Induction of labour (IOL) is a common obstetric intervention and the number of
Strengths and limitations of this study

- Large retrospective data set provides an accurate picture of workload and activity in a UK maternity unit and quantifies delay in the induction of labour process.

- Computer simulation model is innovative and transferable with the potential to be customised to individual maternity units' activity.

- This study uses delay as a proxy marker for clinical risk therefore cannot extrapolate to adverse clinical outcomes.

women having IOL is increasing annually, from $20.4 \%$ in $2007-2008$ to $32.6 \%$ in $2017-$ 2018 in England. ${ }^{2}$ The reasons for this are unclear but it has been attributed to the changing demographic of pregnant women, with rising incidence of obesity, advanced maternal age and increasingly complex needs. There is an increasing body of evidence suggesting that labour induction at term is associated with fewer perinatal deaths and fewer caesarean sections ${ }^{34}$ which may have alleviated clinicians' concerns about the risks of induction and moved the balance of risk and benefit to a more intervention-based approach.

However, the rise in induction rate has resulted in increased workload for maternity units, ${ }^{5}$ and the impact of this on patient care is not well understood. National Institute of Clinical Excellence (NICE) guidance on inducing labour acknowledges that IOL can place more strain on labour wards than spontaneous labour. ${ }^{6}$ In a recent Each Baby Counts report by the Royal College of Obstetricians \& Gynaecologists, delay in the process of IOL has been identified as a key contributory factor to stillbirth, early neonatal death and severe brain injury occurring during labour at term. ${ }^{7}$ Studies of the patient experience of 
women undergoing IOL describe a poor experience of care with protracted delays frequently resulting in patient complaints. $^{89}$

Despite this, there is very little published literature regarding factors associated with delay in the IOL process or interventions to reduce delay and mitigate clinical risk. One study suggested that earlier post-term IOL in high-risk women would only modestly increase obstetric workload in a non-tertiary hospital but made no mention of delay in the process. ${ }^{10}$ Another study on the impact of outpatient induction on midwifery workload found no change in self-reported work demand. ${ }^{11}$ One published quality improvement project focused on reducing inpatient stay by improvement of organisational factors and alterations to the process of IOL, such as outpatient induction and change of prostaglandin preparations; however, the sample sizes were small in each quality improvement cycle and the project took time to assess the impact of each intervention. ${ }^{9}$

In view of the sparsity of evidence, there is a clear need to investigate the factors associated with delay in the IOL process and how these can be mitigated. Computer simulation has been used widely to model healthcare situations $^{12}$ as it provides rapid analysis of the impact of interventions that would otherwise be difficult, timeconsuming and expensive to carry out in real life.

Our objective was to identify factors associated with delay in the IOL process and develop interventions to reduce delay using computer simulation.

\section{METHODS}

This study is comprised of two elements-a retrospective cohort study of real-world maternity unit workload in a large UK district general hospital and a computer simulation model based on the results of the cohort study. The methods for each element are described separately here for clarity.

\section{Setting}

Stoke Mandeville Hospital is a large district general hospital in the UK with approximately 5000 births per year at its maternity unit. The structure of the labour ward comprises 10 delivery rooms and 4 observation beds for high-risk patients, as well as a three-bedded Triage unit for women attending the labour ward acutely with either antenatal concerns or labour. In the event of more than three patients attending Triage simultaneously, women may be assessed in delivery rooms on the labour ward. There are 22 antenatal beds and 24 postnatal beds in a single large ward within the maternity unit. Additionally, there is a co-located midwifery-led birth centre adjacent to the labour ward.

The maternity unit has two obstetric theatres, one where elective category 4 caesarean sections are performed on a separate operating list with separate staffing to emergency operative obstetric procedures. Emergency operative obstetric procedures, including category 3 emergency caesarean sections, are performed by the on-call team and staffed by labour ward midwives.

The maternity unit staffing structure is to have nine midwives on labour ward at all times of day with the ability to increase this by two additional on-call midwives if the workload is high. One of these midwives acts as the labour ward coordinator and one coordinates the Triage unit, while the remaining seven midwives provide one-to-one care in labour for women on both the labour ward and the co-located birth centre. The antenatal and postnatal ward staffing structure is to have seven midwives in hours and five midwives out of hours. Obstetric staffing comprises one consultant who is non-resident out of hours, two resident middle-grade registrars and one resident junior doctor at all times.

In the unit, planned IOLs are booked in antenatal clinic, allowing a maximum of five inductions per day. Women undergoing IOL are admitted to the antenatal ward at 08:00 where they receive prostaglandin E2 (PGE2) for cervical ripening. Primiparous women receive a PGE2 vaginal pessary for 24 hours, followed by a maximum of two PGE2 tablets given at 6-hour intervals. Multiparous women who have had a previous vaginal delivery receive a maximum of two PGE2 tablets given vaginally at 6-hour intervals. Multiparous women who have had a previous caesarean delivery are induced on the labour ward using a maximum of one PGE2 tablet. Patients having IOL are transferred to the labour ward if active labour commences, spontaneous rupture of membranes occurs or when the cervix is deemed favourable enough for artificial rupture of membranes (ARM). ARM is only performed on labour ward and intravenous oxytocin is commenced immediately afterwards in primiparous women and after 2 hours in multiparous women who do not contract after ARM.

\section{Design of retrospective cohort study}

The retrospective cohort study was undertaken to provide an initial baseline analysis of real-world maternity unit workload and to quantify delay in the IOL process.

Anonymised electronic hospital records of all patient admissions in 2018 to the maternity unit were searched for the data described in box 1 . No patient identifiable information was used and this information is routinely collected as part of maternity clinical care for all women.

Additionally, the number of antenatal presentations to labour ward via Triage per day and the number of category 3 caesarean sections per day were recorded, as these also contribute to the labour ward workload. Incomplete data were available for three records therefore these were excluded from the analysis but there were no exclusion criteria.

In this study, IOL was defined as any labour started artificially by any means in the absence of spontaneous labour. This included women electing to have induction with oxytocin for pre-labour rupture of membranes in the absence of contractions but excluded women receiving oxytocin for augmentation of spontaneous labour where contractions were present. IOL was defined as booked or 


\section{Box 1 Maternity unit data variables}

Date and time of admission to hospital

- Parity-primiparous/multiparous.

- Type of onset of labour-spontaneous/induction/caesarean section before labour.

- Indication for induction.

- Booked or emergency induction.

- Medications used for induction.

- Length of time waiting for transfer from antenatal ward to labour ward.

- Length of labour.

Date and time of delivery.

- Mode of delivery-spontaneous vaginal delivery/operative vaginal delivery/caesarean section.

- Number of postnatal days in hospital.

Date and time of discharge from hospital.

emergency depending on their indication and method of arranging the time and date of IOL. Booked inductions were defined as those arranged as a planned intervention via the established booking system in antenatal clinic as described in the Settings section of this paper. Emergency IOL was defined as unplanned or planned with a 24-hour window and arranged directly through the antenatal ward or labour ward. Outpatient induction is not offered at this maternity unit and therefore there are no data for this. Within the electronic hospital patient maternity records, length of labour was defined from diagnosis of active labour according to local hospital guidelines to time of birth in women labouring spontaneously and from ARM and/or commencing of oxytocin infusion in women having induced labour.

These data were then used to quantify delay in the IOL process. Delay in the IOL process was defined as the length of time awaiting transfer to the labour ward from the antenatal ward once a decision had been made for transfer after all cervical priming with prostaglandin had taken place or rupture of membranes had occurred. The study aimed to quantify the length of time that women having IOL were delayed and the number of women experiencing delay. The authors considered that a delay of up to 12 hours should be the benchmark as this represents the length of a typical staffing shift in the maternity unit and allows for the turnover of staff to address workload capacity issues.

The study aimed to quantify staffing shortfall per 24-hour period and staffing levels were assessed using the Birthrate Plus (BR+) system. ${ }^{13} \mathrm{BR}+$ is a nationally endorsed planning tool which allows calculation of the number of midwives required on a labour ward, accounting for both demand and case mix. BR + calculates the number of midwives required by adding up the total time mothers spend on the labour ward and multiplying each of five categories of mother according to interventions received during labour by a multiplier, allowing for increased midwife support for higher acuity labours. This produces a 'Workload Index' which describes the total midwife staffing recommended for coping with the caseload mix. When compared with actual staffing, BR + allows calculation of the staffing shortfall, expressed as the number of midwives short for the case mix described over a given period of time.

\section{Statistical analysis of retrospective cohort maternity unit workload data}

Simple univariate linear regression analysis was performed to assess significant associations between delay in IOL and potential causative factors. The unit of analysis for the linear regression was a week and the outcome assessed was the number of IOL delayed by more than 12 hours within that week-this was selected in preference to single days to allow for the natural variation of workload within any given 24-hour period. Linear regression was selected to assess the continuous variable of number of women delayed per week. Causative factors were selected by author consensus on the basis of their impact on patient flow of workload through the unit. Clinical factors were also considered but this work is not presented here for conciseness.

\section{Design of Maternity Unit Management SIMulation computer simulation model}

The computer simulation was designed to create a computerised artificial maternity unit with simulated patients attending the unit that matched the real-world workload of Stoke Mandeville Hospital as closely as possible, including the creation of simulated delay in patients having IOL. The simulation was named MUMSIM (Maternity Unit Management SIMulation). By building this, the authors were able to plan and test interventions within the simulated unit by changing set parameters and assess their impact on the predicted patient delay.

\section{Simulation model description}

MUMSIM is a computerised simulation of the maternity unit at Stoke Mandeville Hospital, Buckinghamshire National Health Service (NHS) Trust, written in the Python programming language. The model simulates pregnant women arriving at the hospital at random, sampled from a distribution modelled on the real-world data observed at Stoke Mandeville Hospital, collected in the retrospective cohort study. These real-world data are further described in the online supplemental file.

These simulated patients are simulated to go through the labour experiences that are observed in real life, including IOL and spontaneous labour, transferring through the simulated antenatal ward, labour ward and postnatal ward. The individual labour experience is generated at each step in the process within numerical bounds and likelihoods ascertained from the cohort study (for example, range of lengths of active labour and likelihood of particular lengths). For example, the realworld data describe the number of women spontaneously labouring per day with a $40 \%$ probability that between four and sixwomen will present in spontaneous labour 
on any given day and a $16 \%$ probability that a woman in spontaneous labour will present between 08:00 and 12:00. The simulation therefore generates women in spontaneous labour arriving to the simulated maternity unit with a frequency and at a time that corresponds to these probabilities. The simulation also takes into account the longer labour lengths of primiparous women and induced labours and these variables are programmed into the simulation to match the real-world data ranges of lengths of labour by parity and type of onset of labour.

The current state of the simulation, including bed usage and midwife staffing availability, affects the flow of the patients through their experiences. For example, if the simulated labour ward beds are full, a simulated induction would not be transferred to the labour ward, thus creating delay with the simulation.

The induction process used in the unit is described in the Settings section of this paper and it required close replication in our simulation. Data on each step of this process were collected from the cohort study, and used to generate range and frequency for the time scales of each step in the process. This included the likelihood at any time of a step ending in an alternative outcome such as emergency caesarean section. This is then reflected in the MUMSIM model.

\section{Simulation calibration}

The simulation moves forward in 1-minute intervals and an array of data is collected at each interval, allowing production of detailed data for each simulated patient journey through the unit. The data are then output to spreadsheet-compatible formats. This allows users to see when problems arise such as when patients having IOL are delayed waiting to go to the labour ward.

MUMSIM was validated against the data collected in the retrospective cohort study to confirm accurate representation of the real maternity unit. For example, the number of women presenting within a certain time period in the simulation mirrors the ranges and total numbers seen within the real-life data collected. This was validated by running the simulation repeatedly, and then comparing the simulated data with real-life data. CIs of the proportion of the whole were calculated for the reallife data and the simulation was deemed validated when the simulated output fell between the real-world data CIs.

\section{Simulated interventions}

Once validated, MUMSIM was used to test the outcomes of interventions by changing the simulation parameters and observing the impact on the target metric of women waiting more than 12 hours for transfer from antenatal ward to labour ward. The interventions tested are detailed in box 2.

These interventions were chosen by consensus between the authors as representing modifiable parameters that could be altered in real life within our unit, while recognising that this is not an exhaustive list. Interventions such as outpatient induction with balloon catheters were not
Box 2 Interventions tested using Maternity Unit Management SIMulation computer simulation to reduce delay in induction of labour process

1. Allow maximum of two primiparous booked inductions per day.

2. Allow a maximum of four booked inductions per day and distribute inductions throughout the week to reduce variation in number of inductions per day.

3. Change from slow release 24-hour prostaglandin pessary to 6-hour prostaglandin tablet or gel for primiparous women.

4. Change start of induction from 08:00 to 20:00.

5. Increase midwifery staffing to eliminate shortfall.

- One extra midwife during working hours 08:00-17:00.

- One extra midwife at all times.

- One extra on-call midwife.

- Two extra midwives during working hours 08:00-17:00.

- Two extra midwives at all times.

- Two extra on-call midwives.

tested due to the absence of real-world data that could accurately inform the simulation in its current design. All possible combinations of interventions were tested in the simulation but the authors have presented the most salient findings in this paper for conciseness.

Each simulation run represents the simulated maternity unit over a period of 6 months and each intervention was tested with 30 simulation runs. Each run of the simulation has an individual seed number, which creates a different set of patients, presentations and time scales for each seed. This allows the simulation to be run several times to confirm it accurately reflects the cohort data, as each seed will be different and reduces the possibility that the result of any single run is an outlier by chance. Any individual seed can be run with changes to the variable (simulated interventions) to accurately model any effect. As a result, each run of the simulation can be 'paired' to any subsequent run with an intervention-the exact same set of simulated patients will 'arrive' at the unit but the only change will be the intervention and this allows exact comparison of the effect of the intervention.

The measured outcome from each simulation run was the percentage of simulated patients having IOL who experienced delay of more than 12 hours over a simulated 6-month period with the denominator being all simulated patients having IOL. As each individual simulation run starts with an individual seed set of patients, each run generates a different final percentage outcome measurement. After 30 simulation runs, the percentages are pooled to derive an overall mean outcome measurement and SE of the mean and to allow normality testing and statistical analysis - thereby $\mathrm{n}=30$ for the baseline and each tested intervention.

\section{Statistical analysis of computer simulation}

Anderson Darling and Shapiro Wilk normality tests were used to confirm log-normal distribution of the simulation run data. Paired t-tests were used to compare simulation 
Table 1 Results-type of onset of labour by parity

\begin{tabular}{lcccc}
\hline & No of primiparous women & No of multiparous women & Total \\
\hline Spontaneous labour & 1104 & & 1459 & 2563 \\
Induction of labour (IOL) & 797 & Emergency IOL & Booked IOL & Emergency IOL \\
& Booked IOL & 281 & 476 & 242 \\
& 516 & & 641 & 851 \\
Pre-labour caesarean & 210 & & 2818 & 4929 \\
\hline Total & 2111 & & & 8518 \\
\hline
\end{tabular}

runs to assess for significant differences. In all cases, results were considered significant at $\mathrm{p}<0.05$.

\section{Patient and public involvement}

No patients were involved in this retrospective analysis of anonymised routinely collected hospital data.

\section{RESULTS}

There were 4932 births at Stoke Mandeville Hospital, Buckinghamshire NHS Trust in 2018, of which 3 records were incomplete and were excluded from the analysis. Fifty-two per cent of women $(\mathrm{n}=2565)$ laboured spontaneously with $31 \%$ having IOL $(\mathrm{n}=1515)$ and $17 \%$ having a caesarean section before labour $(\mathrm{n}=851)$. Of the women having IOL, 53\% were primiparous $(\mathrm{n}=797)$ and $47 \%$ were multiparous $(n=718)$. These results are summarised in table 1.

Of the women having IOL, $65 \%$ had a planned booked induction $(\mathrm{n}=992)$ and $35 \%$ had an unplanned emergency induction $(n=523)$. The indications for emergency induction included prolonged rupture of membranes without spontaneous labour, meconium-stained liquor or a new diagnosis of an obstetric complication such as pre-eclampsia or fetal growth restriction requiring urgent delivery. The median length of labour was 9 hours for primiparous women and 3 hours for multiparous women and further data on this are available in the online supplemental file.

Of the women having IOL, $42 \%$ of women were delayed more than 6 hours awaiting transfer to labour ward with $33 \%$ delayed more than 12 hours, 20\% delayed more than 24 hours, $11 \%$ delayed more than 48 hours and $1.3 \%$ delayed more than 72 hours.

The results of the linear regression analysis are shown in figure 1A-H. Delay of more than 12 hours was significantly associated with the total number of labouring women $(p=0.008)$, the total number of IOLs $(p=0.041)$ and the number of booked inductions of labour $(p=0.009)$. There was no significant association between delay and the number of women in spontaneous labour, the number of emergency inductions of labour, staffing shortfall, antenatal presentations to the labour ward or category 3 emergency caesarean sections.

The MUMSIM computer simulation was used to test the impact of interventions to reduce delay in the IOL process of more than 12 hours by changing the parameters of the simulation and comparing with the baseline runs. The interventions tested are detailed in the methodology and the results of the simulation runs are shown in figure 2. Results were considered significant at statistically significant at $\mathrm{p}<0.05$ and are marked on the Figure with asterisks.

As shown in figure 2A, Intervention $\mathrm{C}$ of changing from slow release 24-hour prostaglandin pessary to 6-hour prostaglandin tablet or gel for primiparous women reduced delay by $4 \% \quad(\mathrm{p}<0.0001)$ compared with the baseline simulation. No other single intervention reduced delay significantly.

As shown in figure 2B, combined interventions including Intervention $\mathrm{C}$ significantly reduced delay compared with the baseline simulation $(\mathrm{p}<0.0001)$. No other combination of interventions significantly reduced delay compared with the baseline simulation. When compared with Intervention $\mathrm{C}$ alone, the combined Intervention $\mathrm{A}+\mathrm{C}$ showed a further $1.2 \%$ reduction in delay $(p<0.0487)$. No other combination of interventions significantly reduced delay compared with Intervention C alone.

As shown in figure 2C, all additional staffing interventions significantly reduced delay compared with the baseline simulation $(\mathrm{p}<0.0001$ in all cases). Intervention E1 of one extra midwife during working hours of 08:00-17:00 reduced delay by $7.7 \%$, while Intervention $\mathrm{E} 2$ of one extra midwife at all time reduced delay further by $11.7 \%$. Intervention $\mathrm{E} 3$ of one extra midwife available on call reduced delay to a lesser degree by $6.5 \%$. Further reductions in delay were observed with the same patterns of staffing but with two extra midwives in Interventions E4 of 15.2\%, E5 of $17.9 \%$ and $\mathrm{E} 6$ of $10.8 \%$.

\section{DISCUSSION}

Our study is the first to quantify the incidence of delay in the IOL process and shows that delay is a common occurrence. The induction rate in our unit of $30 \%$ is consistent with the national rate of $32.6 \%$. Our findings demonstrate that delay is significantly associated with the total number of labouring women and booked IOL but not emergency IOL, spontaneously labouring women or staffing shortfall.

The MUMSIM computer simulation suggests that reduction in delay within our unit is possible with simple cost-neutral interventions to the IOL pathway, both in the 
A

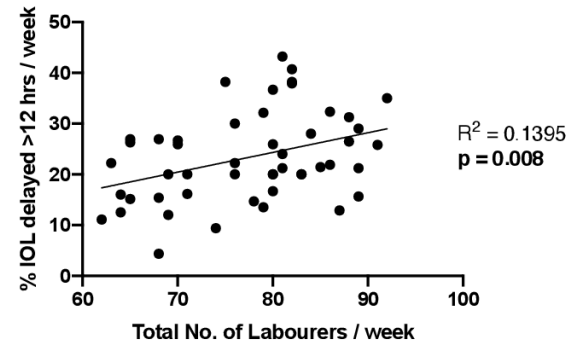

C

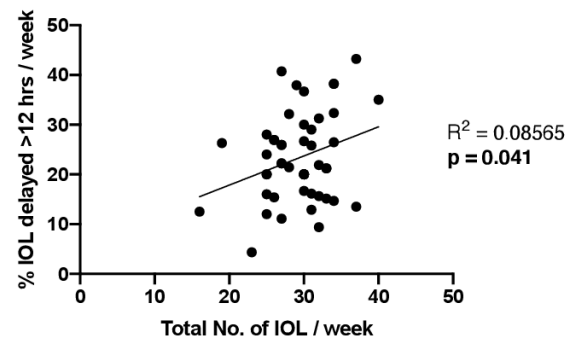

E

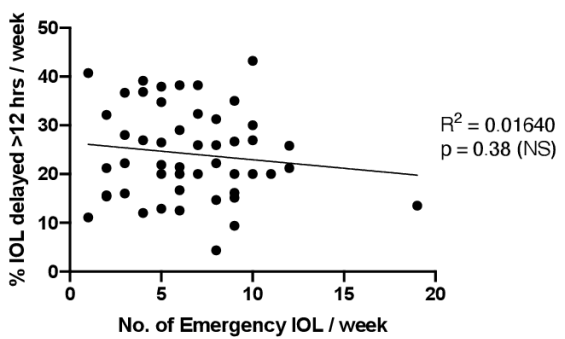

G

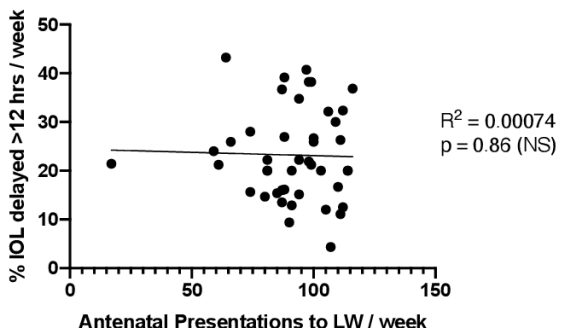

B

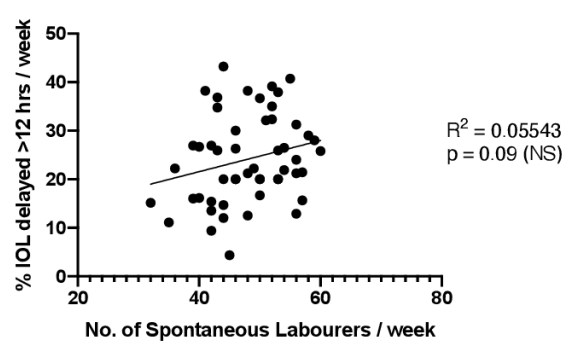

D

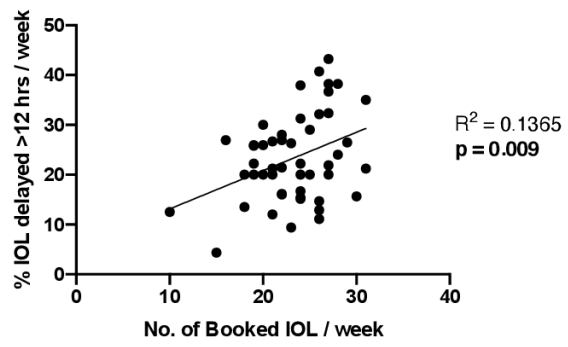

F

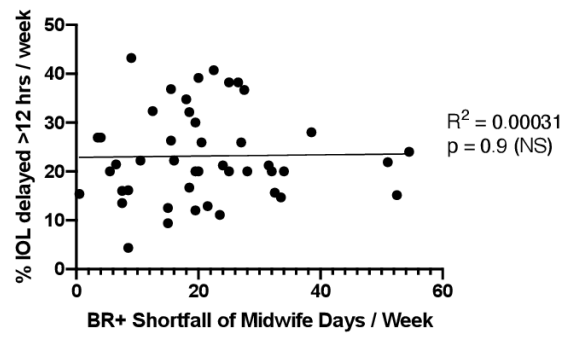

$\mathrm{H}$

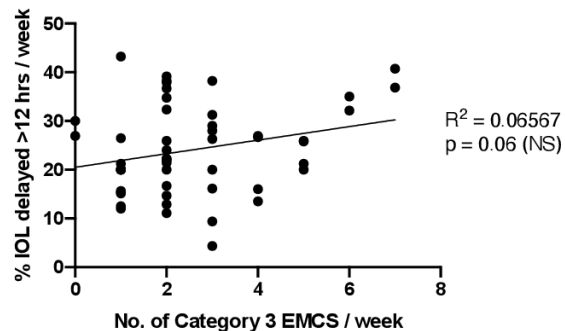

Figure 1 Linear regression analysis of factors associated with delay in the IOL process. The unit of analysis is the number of women having IOL per week experiencing delay of more than 12 hours awaiting transfer from antenatal ward to labour ward versus (A) total number of labourers per week; (B) number of spontaneous labourers per week; (C) total number of IOL per week; (D) number of booked IOL per week; (E) number of emergency IOL per week; (F) Birthrate Plus (BR+) shortfall of midwife days per week; (G) antenatal presentation to labour ward (LW) per week; $(H)$ number of category 3 emergency caesarean sections (EMCS) per week. IOL, induction of labour; NS, not significant.

booking process and in the method of induction used. A significant reduction in delay was observed by changing from slow release 24-hour prostaglandin pessary to 6-hour prostaglandin tablet or gel for primiparous women. The simulation does take into account the longer labour of primiparous women and this variable is programmed into the simulation, meaning that although this intervention makes the initial cervical priming phase of induction analogous between primiparous and multiparous women, the subsequent active labour phase is still differentiated and therefore describes two separate pathways from this point in the simulation afterwards. This therefore does not make all women in the simulated data effectively multiparous and retains differential to allow assessment of the intervention. Cumulative benefit of combining this intervention with limiting the number of primiparous inductions per day to two was also observed. This suggests that there may be benefit for the single intervention of 
A

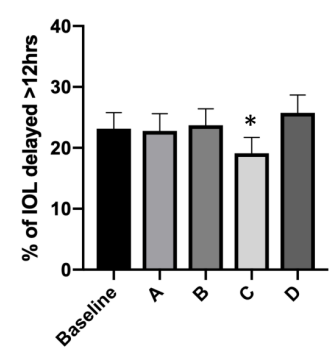

B

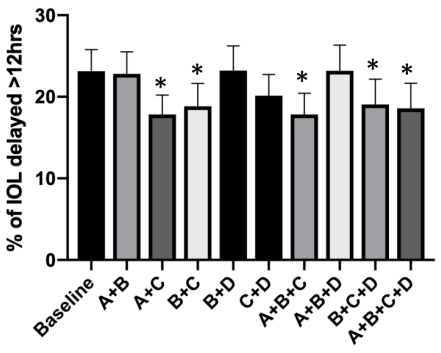

Interventions

A. Allow maximum of two primiparous booked inductions per day

B. Allow a maximum of 4 booked inductions per day and distribute inductions throughout the week to reduce variation in number of inductions per day

C. Change from slow release 24 hour prostaglandin pessary to 6 hour prostaglandin tablet or gel for primiparous women

D. Change start time of IOL from $0800 \mathrm{~h}$ to $2000 \mathrm{~h}$

E. Increase midwifery staffing to eliminate shortfall

C

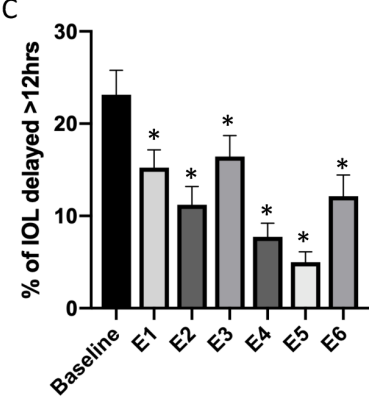

\begin{tabular}{|l|}
\hline Staffing Interventions \\
\hline $\begin{array}{l}\text { E1-One extra midwife during } \\
\text { working hours 0800-1700h }\end{array}$ \\
\hline E2-One extra midwife at all times \\
\hline E3-One extra On Call midwife \\
\hline $\begin{array}{l}\text { E4-Two extra midwife during } \\
\text { working hours 0800-1700h }\end{array}$ \\
\hline E5-Two extra midwife at all times \\
\hline E6-Two extra On Call midwife \\
\hline
\end{tabular}

Figure 2 MUMSIM computer simulation analysis of interventions tested to reduce delay. (A) Single interventions; (B) combined interventions; (C) staffing interventions. IOL, induction of labour; MUMSIM, Maternity Unit Management SIMulation,

limiting the number of primiparous inductions per day to two for units currently not using a slow release 24-hour prostaglandin pessary. All interventions with additional staffing significantly reduce delay and this highlights the importance of staffing in managing obstetric workload safely.

The list of interventions described was chosen by consensus between the authors as representing modifiable parameters that could be altered in real life within our unit and it is acknowledged that there are many more modifiable variables that may contribute to delay in the IOL process and thus scenarios that could be tested using the MUMSIM computer simulation. For example, there is scope for further work using MUMSIM to test the role of outpatient induction using balloon catheters, although the absence of real-world data from our unit to map the patient pathway within the simulation meant that this was not possible in this study. One limitation was lack of robust data on postnatal ward occupancy which can sometimes cause outflow delay from labour ward so although it was calibrated within the simulation, it was not possible to assess for an association by linear regression from the real-world data.
This is a novel study underpinned by a large data set which provides an accurate picture of workload and activity in our unit. The authors recognise that individual maternity units may have different IOL protocols, workload and rate of delay, therefore interventions demonstrated to be of benefit in one unit may not be widely applicable to all. While the interventions described in our study may not be fully generalisable, the MUMSIM computer simulation model is innovative and transferable with the potential to be customised to individual maternity units' activity. Simulations have the additional benefit of engaging clinicians and healthcare leaders in sharing and testing ideas for improvement without fear of failure. One limitation of this study is that it uses delay as a proxy marker for clinical risk and cannot extrapolate to adverse clinical outcomes. Another limitation is that the study does not yet have sufficient follow-up real-world data to confirm the effect of the interventions suggested by the simulation, although further work is ongoing to demonstrate evidence of confirmed benefit in real life. The reason for this is that the nature of fluctuations within the week to week pressure on the units means that a significant period of time of at least 6 months is needed to see the true effect of an intervention. This is a limitation, but also highlights the need for simulation-testing individual interventions in the real world would take 2 years to test four interventions alone.

Our analysis shows that planned obstetric workload of booked IOL is associated with delay rather than the unpredictable workload of women in spontaneous labour or emergency IOL. This suggests that regulating the planned obstetric workload has the potential to improve workflow and reduce delay and thereby clinical risk. Induction may be perceived by clinicians as reducing the risk of adverse perinatal outcome for an individual patient; however, creating excess workload for a maternity unit may increase the cumulative risk for all the women being induced because of capacity. This makes a strong argument for avoiding further increases in the rate of IOL as it may exacerbate delay. Elective induction at 39 weeks has been proposed as a potential strategy to reduce adverse perinatal outcome ${ }^{14}$ but paradoxically may exceed the workload capacity of a maternity unit creating additional delay and increasing the risk of avoidable intrapartum harm. Further research using MUMSIM computer simulation would help quantify the impact of such a policy on maternity units' workload capacity.

Although delay is not associated with staffing shortfall, there is a marked reduction in delay in our simulation with additional staffing. This suggests that delay is unlikely to be due to short-term fluctuations in staffing but rather that it represents a fixed constant within maternity units. Increasing staffing levels therefore forms part of the solution to reducing delay by increasing workload capacity. There is widespread recognition that there is a national shortage of midwives in the UK so increasing staffing levels may be an unrealistic intervention to pilot in maternity units already short staffed. Nevertheless, 
our findings demonstrate that some patterns of staffing are more effective at reducing delay than others and this has cost implications for maternity units considering increasing staffing levels.

\section{CONCLUSION}

Our study demonstrates that many women experience significant delay during the IOL process and the causative factors include the planned obstetric workload of booked induction. Use of a novel computer simulation, MUMSIM, can predict the potential impact of interventions to reduce delay quickly and at zero cost. Further research is needed to ascertain the impact of further rises in the rate of IOL on workload capacity for maternity units, given the potential impact on clinical risk.

\section{TWEETABLE ABSTRACT}

Delay during IOL is common and associated with clinical risk. Computer simulation may help plan interventions to reduce delay.

Contributors KR and JC designed and performed the study and co-wrote the manuscript. IH wrote the computer simulation. RD provided advice on statistical analysis. AR provided access to data collection.

Funding The authors have not declared a specific grant for this research from any funding agency in the public, commercial or not-for-profit sectors.

Competing interests None declared.

Patient consent for publication Not required.

Ethics approval Following discussion with our local research committee, ethics approval was not deemed to be required for a retrospective cohort study of routinely collected anonymised maternity data at our institution.

Provenance and peer review Not commissioned; externally peer reviewed.

Data availability statement All data relevant to the study are included in the article or uploaded as supplemental information.

Supplemental material This content has been supplied by the author(s). It has not been vetted by BMJ Publishing Group Limited (BMJ) and may not have been peer-reviewed. Any opinions or recommendations discussed are solely those of the author(s) and are not endorsed by BMJ. BMJ disclaims all liability and responsibility arising from any reliance placed on the content. Where the content includes any translated material, BMJ does not warrant the accuracy and reliability of the translations (including but not limited to local regulations, clinical guidelines, terminology, drug names and drug dosages), and is not responsible for any error and/or omissions arising from translation and adaptation or otherwise.

Open access This is an open access article distributed in accordance with the Creative Commons Attribution Non Commercial (CC BY-NC 4.0) license, which permits others to distribute, remix, adapt, build upon this work non-commercially, and license their derivative works on different terms, provided the original work is properly cited, appropriate credit is given, any changes made indicated, and the use is non-commercial. See: http://creativecommons.org/licenses/by-nc/4.0/.

ORCID iD

Katherine Robertson http://orcid.org/0000-0002-4414-5186

\section{REFERENCES}

1 NHS. NHS maternity Staties 2017-2018: summary report, 2018.

2 Humphrey T, Tucker JS. Rising rates of obstetric interventions: exploring the determinants of induction of labour. $J$ Public Health 2009;31:88-94.

3 Middleton P, Shepherd E, Crowther CA, et al. Induction of labour for improving birth outcomes for women at or beyond term. Cochrane Database Syst Rev 2018;294.

4 Grobman WA, Rice MM, Reddy UM, et al. Labor induction versus expectant management in low-risk nulliparous women. $N$ Engl J Med 2018;379:513-23.

5 Allen M, Thornton S. Providing one-to-one care in labour. Analysis of 'Birthrate Plus' labour ward staffing in real and simulated labour ward environments. BJOG 2013;120:100-7.

6 NICE. Inducing labour - NICE Clinical guideline [CG70], 2008.

7 Royal College of Obstetricians and Gynaecologists. Each baby counts: 2018 progress report. London: RCOG, 2018.

8 Shetty A, Burt R, Rice P, et al. Women's perceptions, expectations and satisfaction with induced labour--a questionnaire-based study. Eur J Obstet Gynecol Reprod Biol 2005;123:56-61.

9 O'Dwyer S, Raniolo C, Roper J, et al. Improving induction of labour - a quality improvement project addressing Caesarean section rates and length of process in women undergoing induction of labour. BMJ Qual Improv Rep 2015;4. doi:10.1136/bmjquality.u203804.w4027. [Epub ahead of print: 09 Sep 2015].

10 Green B, Howat P, Hui L. The predicted clinical workload associated with early Post-term surveillance and inductions of labour in South Asian women in a non-tertiary hospital setting. Aust N Z J Obstet Gynaecol 2020.

11 Turnbull D, Adelson P, Oster C, et al. The impact of outpatient priming for induction of labour on midwives' work demand, work autonomy and satisfaction. Women Birth 2013;26:207-12.

12 Katsaliaki K, Mustafee N. Applications of simulation within the healthcare context. J Oper Res Soc 2011;62:1431-51.

13 Ball JA, Washbrook M. Birthrate plus: a framework for workforce planning and decision making for midwifery services. Hale, Cheshire, UK: Hochland \& Holland, Books for Midwives Press, 1996.

14 Sinkey RG, Lacevic J, Reljic T, et al. Elective induction of labor at 39 weeks among nulliparous women: the impact on maternal and neonatal risk. PLoS One 2018;13:e0193169. 